\title{
Observando las finanzas como una red de observaciones: Un comentario a Esposito
}

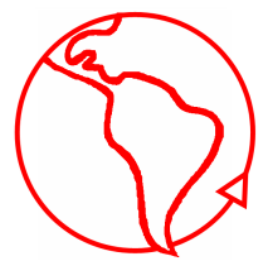

\author{
Observing Finance as a Network of Observations: Comment on \\ Esposito
}

David Stark

Departamento de Sociología, Universidad de Columbia, Estados Unidos

\begin{abstract}
RESUMEN
El presente ensayo presenta una contribución a la teoría de la observación mediante un comentario al artículo de Esposito: "Circularidades económicas y observación de segundo orden: La realidad de las calificaciones." La pregunta clave de dicho artículo puede ser resumida de la siguiente manera: ¿Cómo se puede calcular en el tercer grado keynesiano (intentando determinar lo que la opinión promedio considera como la opinión promedio) en condiciones de circularidad diabólica (cuando la incertidumbre sobre el futuro se genera por las tentativas de predecir el futuro)? Esposito responde que las calificaciones constituyen un punto fijo de referencia, no porque sean exactas, sino porque son muy visibles. La segunda mitad del presente artículo es en sí misma una observación de segundo orden. Utiliza otro punto de vista (el de la teoría de la observación) para reinterpretar mi investigación etnográfica y analítica previa sobre redes en las finanzas.
\end{abstract}

Palabras Clave: Teoría de la Observación; Redes Atencionales; Modelos Financieros; Reflexividad; Evaluación

\section{ABSTRACT}

This essay contributes to observation theory by commenting on Esposito's paper, "Economic Circularities and Second-Order Observation: The Reality of Ratings." The key question of that paper is summarized as: How does one calculate in the Keynesian third degree (attempting to ascertain what the average opinion considers as the average opinion) under conditions of diabolical circularity (when uncertainty about the future is generated by attempts to predict the future)? Esposito answers that ratings provide a fixed point of reference not because they are accurate but because they are highly visible. The second half of the paper is itself a second-order observation. It uses another viewpoint (that of observation theory) to reinterpret my earlier ethnographic and network analytic research on finance.

KEYWORDS: Observation Theory; Attention Networks; Financial Models; Reflexivity; Valuation

\section{INTRODUCCIÓN ${ }^{*}$}

"Se pueden observar muchas cosas con solo mirar" (Yogi Berra). Esta cita, perteneciente a uno de mis filósofos Yankees favoritos, debería ser el lema de los etnógrafos. Mirar, estar allí, in situ, con ojos y oídos abiertos, en una actitud de curiosidad, sin saber de antemano lo que se busca

\footnotetext{
* [N. del T.] Traducido del inglés por Hugo Cadenas, con autorización del autor. Una versión previa de este artículo apareció en: Sociologica, N², 2013.
}

REVISTA MAD - UNIVERSIDAD DE CHILE

Revista del Magíster en Análisis Sistémico Aplicado a la Sociedad

Facultad de Ciencias Sociales, Departamento de Antropología.

Universidad de Chile

www.revistamad.uchile.cl 
pero estando preparado para reconocerle cuando se lo encuentra - esta sigue siendo una de las mejores técnicas para la recolección de datos. Pero, por supuesto, los etnógrafos no tienen el monopolio sobre la observación de otros. De hecho, una de las premisas fundamentales de la teoría de la observación, tal como la ha formulado Niklas Luhmann y desarrollado Elena Esposito (2011, 2012), es que la sociedad se constituye por este proceso de observación mutua. En un trabajo anterior, extraordinariamente provechoso, Esposito propuso la idea que "el verdadero objetivo y la función del mercado (...) es proporcionar una arena para la observación mutua de observadores" (Esposito 2012: 10). En el artículo que discutimos aquí, ella expande esta idea a fin de aplicar la teoría de la observación al campo de las finanzas y, en particular, al estudio de las calificaciones crediticias.

Los lectores que no son de Norteamérica podrían tener la impresión de que el 'filósofo Yankee' de mi frase inicial es un señor de Nueva Inglaterra, parecido quizás al poeta Robert Frost. Aunque algunas de las declaraciones de Berra realmente pueden leerse poéticamente, Yogui no era un hacendado. Él era, en efecto, un Yankee-un Yankee de Nueva York- uno de los jugadores de béisbol más excepcionales, el cátcher de un equipo dos veces campeón que puso en el campo a algunos de los mayores atletas de dicho deporte. Además de sus talentos en el béisbol, Yogi Berra era un consumado y ocurrente bromista, famoso por comentarios lacónicos como: "esto es como un déjà $v u$ otra vez," "nunca contesto una carta anónima," y "si te encuentras un tenedor en el camino, tómalo." Truismos, ciertamente, pero a veces más paradójicos y siempre, curiosamente, reveladores. Aunque su sucinta explicación de la inflación -"una moneda de cinco centavos ya no vale lo que una de diez" - podría ser su contribución más contundente a la teoría de las finanzas, emplearé varias de sus otras observaciones como un dispositivo para replantear y discutir el excelente artículo de Esposito.

Comenzaremos con el argumento de Esposito respecto a que se equivocan aquellas nociones de la economía que asumen que esta tiene un interior y un exterior. No hay ninguna posición desde la cual pueda observarse la economía desde fuera. Hay perspectivas diferentes, desde luego, pero ninguna contempla a la economía desde una posición externa a ella. Se pueden observar muchas cosas con solo mirar, como dice mi amigo Yankee. Cuando uno mira, ve que las observaciones son parte del mundo, son parte de la economía. Estas incluyen a las observaciones de actores cotidianos, así como a las de los economistas -incluso a las de los 
sociólogos. Por estos motivos, Esposito sostiene que el enfoque de la incrustación [embeddedness] -con sus conceptos de economía y sociedadno constituye un punto de partida útil para la sociología económica. Los sociólogos no están fuera de la economía, observándola desde algún ángulo de la sociedad. Para Esposito, la economía no está incrustada en las observaciones. Las observaciones son parte constitutiva de la economía. Concuerdo con esto. Además he sostenido en otra parte (Stark 2009) que ya es tiempo que la sociología económica se mueva más allá de la noción de los mercados incrustados en relaciones sociales, del valor anidado en valores, de la economía apoyándose en algún colchón cultural. En vez de estas redes, nidos y muñecas rusas, una mejor metáfora para la economía sería la de la banda de Möbius -la forma topológica sin interior y exterior.

\section{1. "YA NADIE VA A ESE LUGAR PORQUE VA DEMASIADA GENTE"}

En la segunda parte de su ensayo, Esposito adopta la noción del 'concurso de belleza keynesiano.' En dicho concurso, todos los jueces son también, en un sentido importante, los verdaderos concursantes, puesto que compiten por ver quién puede anticipar, en palabras de Keynes, "lo que la opinión promedio espera que sea la opinión promedio." Yogi Berra no es John Maynard Keynes, pero comprende realmente la diferencia entre un inversionista y un analista técnico ${ }^{*}$, este último es como un fashionista en el restaurante o en el circuito de clubes nocturnos que presta atención a la música o a la comida solo en lo que resulta necesario para el verdadero juego, el cual consiste en prestar atención a la muchedumbre. El objetivo de dicha competencia es: anticipar la cresta y seguir siendo (en sentido figurativo) el primero en la puerta cuando el lugar se llena demasiado, justo antes que 'la música se detenga' (la acción se desploma, la burbuja estalla).

El uso de Esposito del concurso de belleza keynesiano es más sofisticado que un simple análisis técnico. Ella quiere que veamos a las finanzas contemporáneas como si estuvieran casi totalmente desconectadas de la materialidad. El predominio de los derivados y la prevalencia de la circularidad (nótese que los keynesianos hablan de "especularidad", v. Dupuy 1989) da lugar a una situación en la que "se abandona cualquier referencia a criterios 'objetivos' (del mundo exterior) y uno se aventura en el campo de las opiniones y las estructuras sociales" (Esposito 2014: 7).

\footnotetext{
" [N. del T.] Se llama "analista técnico" en el mundo financiero a quien analiza los mercados a través de
} gráficos y busca identificar y predecir tendencias en el futuro. 
Para Esposito, la metáfora del concurso de belleza keynesiano "indica que el mundo financiero se guía por criterios precisos, y no arbitrarios, que no tienen nada que ver con la calidad real de los bienes o la solidez de las compañías -o con otros presuntos 'fundamentos' de la economía" (Esposito 2014: 7). No se necesita haber adoptado alguna teoría financiera equivalente a la teoría de la correspondencia o de la verdad para notar que es hiperbólico insistir en que el mundo financiero no tiene nada que ver con la solidez de las compañías. Quisiera concentrarme en cambio en la noción de observación de segundo orden. En este punto el lector debe recordar que actúo con la profundidad filosófica de un jugador de béisbol estadounidense y se me debe perdonar si me confundo con toda esta conversación acerca de las diferencias entre las observaciones de primer y segundo orden luhmannianas, llevadas a los 'terceros, cuartos, y quintos niveles keynesianos' de circularidad. Esposito rotula esta sección como "El concurso de belleza como observación de segundo orden." El objeto de estudio de los sociólogos, aprendemos, no debería ser el de las observaciones de primer orden, porque:

No conducen la dinámica de las operaciones, las cuales se centran más bien en la observación de segundo orden, en la mutua observación de la observación de los otros, y esto ocurre hasta muy altos y aparentemente inextricables niveles de abstracción y circularidad. (Esposito 2014: 7).

Sin frivolidad: me confunde el concepto de observación de "segundo orden". En el pasaje recién citado, la observación de segundo orden es "la mutua observación de la observación de los otros." ¿Puedo observar tu observación? ¿Puedes observar la mía? Si mutuamente nos observamos unos a otros, ¿son estas entonces "observaciones de segundo orden"? Si lo son, ¿cómo? ¿Qué significaría que he observado tu observación? Por supuesto, no puedo estar en tu cabeza; no puedo observar tu percepción. Sin embargo puedo observar tus visiones expresadas o las comunicaciones grabadas sobre tus observaciones.

Imagina, por ejemplo, que eres un analista de valores. Puedo leer tus informes, tus estimaciones sobre las ganancias de un determinado valor. Pero esto no es diferente -como un orden de observación, que es el tema en cuestión- de mirar el teletipo de la bolsa, escuchar a un presidente ejecutivo en una teleconferencia, o leer un informe de la compañía. Ya que no puedo leer tu mente, mi lectura de tu comunicación grabada (tu "observación") es necesariamente una observación de primer orden. Tal vez estoy (obstinadamente) entendiendo mal, pero no veo cómo la "la 
mutua observación de la observación de los otros" es una "observación de segundo orden", cuando en cambio parece tratarse derechamente de un caso de observaciones de primer orden multilaterales.

¿Qué tal si te miro observando? ¿Podría esto ser una "observación de segundo orden"? Observar cómo prestas atención -tu postura, por ejemplo, o tu nivel de atención- me parece trivial. En cualquier caso, todavía sería una observación de primer orden. Quizás una observación de segundo orden se refiere entonces a tu observación sobre mi observación, o la mía sobre la tuya. Este mismo comentario podría ser visto como una meta-observación, por ejemplo, mis observaciones sobre las observaciones de Esposito. Pero sean meta-observaciones o no, de primer o segundo orden, para esta problemática no son diferentes de las observaciones del analista de valores (en efecto, un informe acerca de informes) que habíamos establecido antes como observación de primer orden.

Lo que importa aquí es que estoy de acuerdo con Esposito respecto que algo está ocurriendo particularmente en el campo de las finanzas, lo cual no puede ser capturado con la noción de una observación de primer orden. Precisamente porque este proceso es importante es que creo que el lenguaje de la 'observación' no es el instrumento analítico más apropiado para investigar el problema. Puedo observar tus observaciones, en el sentido de hacer observaciones de primer orden de tus comunicaciones sobre tus observaciones. Pero sería más valioso, (en muchos casos, mucho más valioso) que tu comunicación expresada, el que yo pudiera tener acceso al esquema interpretativo que dio origen a tus observaciones comunicadas. En otras palabras, quisiera tener acceso a tu modelo. En algunos casos es posible que desees revelar tu modelo. Pero en los casos más interesantes, tu modelo (el esquema a través del cual interpretas el mundo y tomas decisiones, incluyendo cómo interpretas tus observaciones sobre mis comunicaciones) está protegido. Si decides no revelarlo, no puede ser observado. Pero podría inferirse. Volveremos luego a esta pregunta sobre el velo de los modelos ocultos.

La pregunta es si Yogi Berra obtiene la última palabra o no, y de ser así, cómo. Tal vez se pueden observar muchas cosas con solo mirar. Tal vez, también, hay muchas cosas que no se pueden observar mirando. Y quizás hay algunas cosas que se pueden observar, pero no mirando. Es decir, podría ser que si yo hubiera estado lo suficientemente atento, habría notado que Yogui trataba de alertarme desde el principio sobre la posibilidad que pudiera existir una diferencia entre observar y mirar. 
2. "El FUTURO NO ES LO QUE SOLÍA SER"

En la tercera parte de su artículo, Esposito extiende el concepto de 'riesgo moral' de un modo fascinante, yendo más allá de la noción de que el seguro produce nuevos riesgos. Para ella, el aspecto más interesante de la circularidad de las observaciones se refiere a la incertidumbre sobre el futuro y el aspecto más preocupante es que los modelos que predicen el futuro pueden, al ser usados, originar un mundo diferente al que se predijo. Esto es una circularidad diabólica: mientras más se sigue una predicción, más se modificarán las condiciones sobre las cuales se ha basado, y con ello el mundo cambia. Ahora podemos entender cuán apropiada es la afirmación de nuestro filósofo de Yankee: "El futuro no es lo que solía ser." Las observaciones sobre el futuro originan futuros diferentes.

Esta es la sección más breve del artículo que discutimos, en parte porque Esposito ha escrito sobre este tema en otra parte y más ampliamente (Esposito 2009, 2012). Esposito señala que cualquier modelo necesitaría hacer suposiciones sobre las acciones de los otros. Las cosas se ponen realmente interesantes, sostiene, cuando los modelos se hacen más sofisticados y comienzan a considerar que los otros no actúan simplemente, sino que actúan sobre la base de modelos (los que asimismo consideran que otros usan modelos, cada uno de los cuales tiene probabilidad de estar basado). Como los modelos se hacen más sofisticados, más potentes, y más capaces de tener en cuenta el riesgo del modelo, los precios se vuelven más volátiles y el sistema en conjunto se hace menos predecible. Es decir la fiabilidad de los modelos contribuye a la imprevisibilidad del sistema:

Bajo estas condiciones, cada pronóstico fiable está destinado a falsearse a sí mismo, porque el futuro reacciona a las expectativas que le son impuestas donde cada pronóstico fiable adicional contribuye a un aumento en la imprevisibilidad del futuro (Esposito 2009: 370).

Estoy lejos de estar familiarizado en materia de la teoría de las probabilidades, por lo que no se debería confiar en mi resumen. Pero en el espíritu de una ocurrencia de Yogi Berra, esto se leería: es probablemente improbable que la improbabilidad dure. 
¿Cómo hace uno entonces para calcular el tercer grado keynesiano (intentando averiguar lo que la opinión promedio considera como la opinión promedio) bajo condiciones de circularidad diabólica (cuando la incertidumbre sobre el futuro es generada por tentativas de predecir el futuro)? Cuando todo está en un movimiento incierto, ¿a qué puedo atar mi algoritmo? Esposito responde que las calificaciones proporcionan tal punto fijo de referencia. Para cumplir esta función, las calificaciones no necesitan ser perfectas. De hecho, su función como punto de referencia, sostiene Esposito, puede ser separada de su función predictiva. Lo que importa es que proporcionan un estándar común, "una referencia compartida y visible, una opinión que está disponible para todos y que todos saben que se sabe" (Esposito 2014: 18). En una situación de invisibilidad generalizada de las observaciones de los otros, no es la exactitud de las calificaciones, sino su alta visibilidad lo que les da valor.

\section{4. "Si NO SABES HACIA DÓNDE VAS, TERMINARÁS EN CUALQUIER OTRO LUGAR"}

El artículo de Esposito me incitó a pensar de nuevo en mi propia investigación sobre las finanzas. No sabía exactamente hacia dónde me dirigía con ese trabajo, pero ahora veo que estoy llegando muy cerca de la teoría de la observación, a pesar de no haber entendido antes la conexión explícita. Por ejemplo, en un artículo reciente (Beunza \& Stark 2012), Daniel Beunza y yo nos hacemos la pregunta: ¿Cómo se enfrentan los operadores financieros a la falibilidad de sus modelos? En particular, cómo lidian con el hecho que, en la identificación de patrones en los mercados, estos mismos instrumentos pueden también cegar al operador de ver algunas cosas. Como instrumentos de percepción -y en efecto, como el propio nervio óptico que nos permite ver, pero que debe producir también un punto ciego- los modelos que revelan también ocultan.

¿Cómo evitan los operadores dicho bloqueo cognoscitivo? La respuesta es que estos aprovechan el hecho que otros operadores observan de una posición estratégica diferente. Los operadores en la oficina de arbitraje de fusiones que estudiamos no podían observar lo que estaba en las pantallas de sus rivales. Es decir, en tanto operador no puedo observar directamente tus observaciones y no tengo acceso a tu modelo. Lo que me gustaría es hacer inferencias razonables sobre tu modelo. Beunza y yo mostramos que, en caso del arbitraje de fusión, los operadores ubican en 
sus pantallas una imagen de 'gráfico diferencial' [spread plot], la cual usan hábilmente como una representación de las visiones agregadas de sus rivales. Cuándo el gráfico diferencial se mueve en una dirección diferente de las propias estimaciones, los operadores pueden preguntar, '¿Qué no estoy viendo?' y hacer correcciones a sus modelos. Mirar el gráfico diferencial es en sí una observación de primer orden. Pero cuando el diferencial se mueve en una dirección diferente a las estimaciones derivadas de mi modelo privado, la triangulación resultante es una observación de segundo orden que me permite hacer inferencias sobre cómo interpretas el mundo, lo cual puede hacerme reflexionar (pensar otra vez) sobre mi propio modelo. Dicho 'modelamiento reflexivo' puede ayudar a un operador individual a evitar el desastre, pero debería venir con una señal de advertencia: cuando el sistema carece de la diversidad necesaria, la interdependencia cognoscitiva puede crear una retroalimentación positiva que genere un desastre en el arbitraje financiero -como los $\$ 2,8$ billones en pérdidas para los arbitrajistas de fusión (incluyendo el equipo que estudiamos) en el intento de fusión de General Electric con Honeywell. Cuando el sistema carece de diversidad de puntos de vista, las mismas prácticas que resultan eficaces para la mitigar el bloqueo cognoscitivo individual pueden conducir a un bloqueo cognoscitivo colectivo de enormes proporciones.

Beunza y yo basamos nuestro argumento en prolongadas observaciones etnográficas de arbitrajistas de fusión que operan con derivados financieros en un importante banco de inversión internacional en Wall Street. El artículo que escribimos fue elaborado a partir de observaciones de una oficina de arbitraje de fusión, en una sala de operaciones comerciales (de hecho, limitando nuestro reporte aún más a lo que ocurrió en una sola mañana). En un artículo posterior, Matteo Prato y yo utilizamos un método muy diferente -un análisis estadístico de 10.933 .662 pares de estimaciones de analistas de valores sobre las ganancias por acción listadas públicamente de empresas en EE.UU- para estudiar los efectos de las estructuras sociales de observación en la evaluación.

Dicho estudio (Prato \& Stark 2013) se basa en el principio de la teoría de la observación relativo a que la valoración depende del punto de vista contingente del observador y de las visiones expresadas por el observado. Los puntos de vista del observador y las visiones observadas están, para nosotros, incrustados en estructuras de redes atencionales evolutivas y bimodales (agentes-activos) que caracterizan a los mercados financieros. Nuestro argumento comienza con una simple pregunta: ¿Qué significa concentrarse en un activo financiero? 
Una manera de pensar esto es como una relación singular entre un actor y el activo. Otra forma, muy popular entre los sociólogos, es pensar en un actor que examina un activo en relación con una categoría abstracta. Nosotros adoptamos una perspectiva diferente: En vez de postular que es la "estructura de la clasificación la que dirige la evaluación" (Zuckerman 2004: 411), sostenemos que es la estructura de la atención la que dirige la evaluación. En el lugar de argumentar que la evaluación está incrustada en categorías socialmente construidas, afirmamos que está moldeada por redes de atención.

Definimos una red atencional como una red evolutiva creada por múltiples agentes que fijan su atención y expresan sus juicios a través de múltiples situaciones. La evaluación, sostenemos, es moldeada por la posición (o punto de vista) de un actor dentro de dicha red atencional. Es decir, como un primer paso, proponemos estudiar la relación entre prestar atención y fijar la atención. Focalizar la atención y fijar la atención no son muy diferentes. Los objetos a través de los cuales se fija la atención son el suelo contra el cual se puede ver la figura. Si como investigadores podemos conocer los otros objetos que un actor tiene en su campo de visión, entonces conocemos el punto de vista a partir del cual se realiza una evaluación.

En la evaluación de una situación focalizada, los actores pueden hacer asociaciones, analogías, y comparaciones con otras situaciones que están presentes en su cartera de atención. En concreto, una característica considerada como sobresaliente para evaluar un problema podría ser reconocida como relevante para otro. Es decir, los problemas a través de los cuales un actor fija su atención moldean las propiedades que son seleccionadas como sobresalientes y dignas de consideración a la hora de evaluar una situación focalizada.

Nos referimos a esto como el efecto de los puntos de vista. Nuestra primera propuesta es que la evaluación es perspectivista: la evaluación de alguien sobre un tema está modelada por su punto de vista; por su contingente cartera de atención. Nuestra hipótesis es, concretamente, que dos actores que evalúan una situación dada frente a una cartera compuesta de manera similar (diferente) de otras situaciones, se encuentran más propensos a converger (diverger) autónomamente en sus interpretaciones de la situación dada.

Los puntos de vista son el primer, pero no el único, paso para el desarrollo de un enfoque de redes de observación de las evaluaciones. Sobre la base de la segunda propiedad relacional de la atención en una red 
de observación bimodal (esto es, relaciones entre los competidores que prestan atención a los mismos problemas del mercado), se espera que haya mayor probabilidad que los actores del mercado se encuentren con las evaluaciones de los competidores que focalizan su atención en los mismos problemas. Cuando dos competidores fijan su atención a través de carteras de problemas muy similares, sus visiones se hacen muy visibles las unas para las otras. Las asociaciones hechas por un actor se hacen evidentes para otro y viceversa. A la inversa, la exposición mutua se vería limitada cuando dos competidores no se ubican en sus respectivos campos de visión, debido a que fijan su atención en diferentes aspectos del mercado.

De este modo, nuestra segunda propuesta relativa al efecto de los puntos de vista, es que la evaluación es doblemente perspectivista: las evaluaciones de los actores no solo se encuentran moldeadas por sus puntos de vista contingentes, dados por sus fugaces carteras de atención, sino también por las visiones de otros, las cuales están moldeadas ellas mismas por sus cambiantes puntos de vista. Por lo tanto, planteamos adicionalmente la hipótesis que mientras más (menos) ocurra que dos actores encuentren las mismas visiones en terceros sobre otras situaciones a las cuales no han estado atentos en conjunto, más convergirán (divergirán) sus interpretaciones de una situación dada.

Probamos estas propuestas en el contexto de los analistas de valores, a quienes podríamos considerar como observadores profesionales. En particular, estudiamos las estimaciones de ganancias para final del año que los analistas de valores hacen sobre las firmas en sus coberturas de cartera. Nuestros resultados apoyan la idea que la posición de un actor en una red de observación -a través del punto de vista y la exposición selectiva a las visiones de los otros- moldea la evaluación.

Nuestros análisis mostraron, en primer lugar, que la estimación de un analista respecto de las ganancias por acción de un valor dado para final de año, se encuentra moldeada por los otros valores en su campo de visión. En términos de la idea de Hill-Popper (2004: 91) respecto que la evaluación ocurre a partir de la "orientación particular de un individuo hacia un objeto de intercambio", encontramos que cuando evalúa un valor dado, un analista no se enfrenta por sí solo a dicho valor. En el lugar de una relación singular -un solo analista frente a un solo valor- encontramos un conjunto más bien multilateral de relaciones. El valor no está solo. Tal como indican nuestros resultados, este se evalúa en términos de otros valores que están en el campo de visión del analista. 
Nuestros análisis demostraron además que las estimaciones de los analistas estaban influenciadas por las visiones de otros analistas con quienes compartían misma la cobertura de valores y que dichos efectos se amplificaban cuando los individuos compartían patrones de atención con las mismas terceras partes. ¿Cómo investiga un analista dado cuando sabe que posee capacidades cognoscitivas limitadas? Nuestra respuesta comenzó con una proposición simple: El analista no está solo. Nuevamente, la relación entre analista y valor no es singular -hay diversos analistas que evalúan el mismo valor, cada uno de los cuales evalúa simultáneamente otros valores. Dadas las limitadas capacidades cognoscitivas individuales, los analistas aprovechan estas relaciones multilaterales. Al igual como la visión del valor focalizado no se moldea solamente por la información sobre aquel valor, sino también por otros valores que dan forma al trasfondo, de este modo sostenemos que la visión del valor focalizado no se moldea solamente por las visiones de otros sobre aquel valor, sino también por las visiones sobre otros valores que no se comparten.

Si mis visiones son moldeadas por mi visión periférica y las tuyas por tu propia visión periférica, en la medida que se influencian mutuamente, podemos decir que mis opiniones son moldeadas, en parte, por tu visión periférica.

Nuestra explicación sociológica sobre las evaluaciones usufructúa de las redes bimodales como método de análisis. Los objetos se ubican dentro de una estructura de red atención dada por actores que los observan y evalúan. Por su parte, los actores también se ubican dentro de una estructura de atención dada por los lazos que los unen a través de los objetos que observan y evalúan. Nótese la peculiar característica de esta red. No hay lazos directos entre los agentes. Ellos no se encuentran próximos entre sí debido a una especie de conexión personal. Su ubicación en el espacio social de la atención -su proximidad o distancia mutua- es una función de lazos formados a través de objetos. En la cartografía de estas redes, trazamos redes socio-cognoscitivas.

Mientras que problemas como el concurso de belleza keynesiano estimulan a los economistas a pensar en la intersubjetividad (Fullbrook 2001), consideramos nuestra adopción del análisis de redes bimodales como un método para estudiar la interobjetividad. 
CONCLUSIÓN: "NO SE TERMINA HASTA QUE SE TERMINA"

La frase de Heinz von Foerster "No vemos lo que no vemos" podría bien haber sido un aforismo de Yogi Berra -en ambos se puede ver que una buena tautología puede ser informativa. ¿Tiene la teoría de la observación un punto ciego? Por supuesto, debe tenerlo. En la medida en que proporciona un lente para ver, debe también ocultar de la observación algún elemento, momento o situación. Toda teoría tiene un punto ciego. En el lugar del singular 'soy un luhmanniano' (o el igualmente singular 'soy un etnógrafo' o 'soy un analista de redes'), lo correcto sería la visión teórica binocular. Frente a la defensa: 'oh, pero seguramente tú, al igual que yo, necesitas una identidad', uno puede contestar que una verdadera identidad es aquella que está con la discrepancia, en la diferencia, dentro de la disonancia. Dante lo expresó de forma ligeramente diferente en el pasaje del Purgatorio de La Divina Comedia: "No fijes tu atención en un solo punto". El mandato de Dante es un buen consejo para abordar el problema de quedarse atrapado en los propios éxitos. Una visión sociológica doble puede ayudar a evitar tal bloqueo cognoscitivo. Por supuesto, la visión doble es una especie de enfermedad, las cosas se desenfocan. Pero 'el foco' puede estar sobreestimado, sobre todo si se trata de una variedad pertinaz.

Tan a menudo oímos el consejo, ya sea para las organizaciones o, por ejemplo, para nuestros estudiantes: '¡enfóquese!' Pero, siguiendo con esta metáfora visual, también hay algo que decir acerca de la importancia de la visión periférica. Es de vital importancia para los atletas. Es una habilidad útil y necesaria para moverse con rapidez junto a otras personas, yendo en direcciones diferentes, como me he percatado navegando desde una línea de metro hacia la otra durante la hora punta en la estación de metro Times Square en Nueva York. Es también esencial para las organizaciones. En entornos de alta incertidumbre, no deberías cerrarte mirando hacia adelante (con una visión doblemente equivocada respecto que el futuro puede ser previsto y que debe estar necesariamente adelante) sino también debes prestar atención al movimiento que ocurre a tu alrededor. La visión periférica toma conciencia de este movimiento.

En la ciencia, así como para las organizaciones, el binocular tiene ventajas. Somos ciegos a nuestros puntos ciegos, nos dice von Foerster. No podemos encontrar un punto de pura exterioridad, como si en una especie de sociología aérea, la objetividad y/o reflexividad fuesen una función de la distancia. Estamos siempre dentro. No hay una teoría social de 
la banda de Möbius. En el lugar de ningún interior/ningún exterior, podemos operar en otra forma topológica -el pliegue estructural- en el interior de más de una comunidad (Vedres \& Stark 2010, De Vaan, Vedres \& Stark 2012). De este modo, podemos aspirar a la reflexividad como una propiedad no de un individuo, sino de una colectividad. ¿Qué es mejor que una observación? Una conversación.RM

\section{BIBLIOGRAFÍA}

Beunza, D. \& Stark, D. (2012). From Dissonance to Resonance: Cognitive Interdependence in Quantitative Finance. Economy and Society, 41(3), 383-417. de Vaan, M., Vedres, B., \& Stark, D. (2012). Game Changer: The Topology of Creativity in Video Game Development. Columbia University Center on Organizational Innovation, Working Paper Series.

Dupuy, J.P. (1989). Common Knowledge, Common Sense. Theory and Decision, 27(12), 37-62.

Esposito, E. (2009). The Certainty of Risk in the Markets of Uncertainty. En W. Hafner \& H. Zimmermann (Eds.), Vinzenz Bronzin's Option Pricing Models: Exposition and Appraisal (pp. 359-372). Berlin/Heidelberg: Springer.

Esposito, E. (2011). The Future of Futures. The Time of Money in Financing and Society. Cheltenham: Elgar.

Esposito, E. (2013). The Structures of Uncertainty. Performativity and Unpredictability in Economic Operations. Economy and Society, 42(1), 102-129.

Esposito, E. (2014). Circularidades económicas y observación de segundo orden: La realidad de las calificaciones crediticias. Revista Mad, 30, 1-24. [En esta edición]

Fullbrook, E. (Ed.) (2001). Intersubjectivity in Economics: Agents and Structures. New York/London: Routledge.

Podolny, J. \& Hill-Popper, M. (2004). Hedonic and Transcendent Conceptions of Value. Industrial and Corporate Change, 13(1): 91-116.

Prato, M. \& Stark, D. (2013). Attention Networks: A Two-Mode Network View on Valuation. Columbia University Center on Organizational Innovation, Working Paper Series.

Stark, D. (2009). The Sense of Dissonance: Accounts of Worth in Economic Life. Princeton, NJ: Princeton University Press.

Vedres, B. \& Stark, D. (2010). Structural Folds: Generative Disruption in Overlapping Groups. American Journal of Sociology, 115(4), 1150-1190.

Zuckerman, E. (2004). Structural Incoherence and Stock Market Activity. American Sociological Review, 69(3), 405-432.

\section{SOBRE EL AUTOR}

David Stark es profesor del Departamento de Sociología de la Universidad de Columbia en Nueva York, Estados Unidos. Doctor en Sociología por la Universidad de Harvard, Estados Unidos. Entre sus áreas de especialización se encuentran: Sociología Económica, Sociología de la Innovación, Democratización y Cambio Organizacional 
en la Europa del Este Postsocialista. Entre sus publicaciones se destacan: This Place, These People: Life and Shadow on the Great Plains (con Nancy Warner) [Columbia University Press. 2013]; The Sense of Dissonance: Accounts of Worth in Economic Life [Princeton University Press, 2009]; Postsocialist Pathways: Transforming Politics and Property in East Central Europe (con László Bruszt) [Cambridge University Press, 1998].

\section{CONTACTO}

Department of Sociology

Columbia University 606 W. 122nd Street

New York, NY 10027

dcs36@columbia.edu

Recibido: Octubre 2013

Aceptado: Enero 2014 